\title{
Best Approximations in Hardy Spaces on Infinite-Dimensional Unitary Matrix Groups
}

\author{
Oleh Lopushansky \\ Faculty of Mathematics and Natural Sciences, University of Rzeszów, 1 Pigonia Street, 35-310 Rzeszów, Poland \\ Correspondence should be addressed to Oleh Lopushansky; ovlopushansky@hotmail.com
}

Received 29 December 2013; Accepted 14 June 2014; Published 6 August 2014

Academic Editor: Detlev Buchholz

Copyright (C) 2014 Oleh Lopushansky. This is an open access article distributed under the Creative Commons Attribution License, which permits unrestricted use, distribution, and reproduction in any medium, provided the original work is properly cited.

\begin{abstract}
We investigate the problem of best approximations in the Hardy space of complex functions, defined on the infinite-dimensional unitary matrix group. Applying an abstract Besov-type interpolation scale and the Bernstein-Jackson inequalities, a behavior of such approximations is described. An application to best approximations in symmetric Fock spaces is shown.
\end{abstract}

\section{Introduction}

Our goal is to investigate a best approximation problem in the quasinormed Hardy space $H_{\chi}^{p}(0<p \leq \infty)$ of complex functions of infinitely many variables. The considered Hardy space is defined on the infinite-dimensional unitary matrix groups $U(\infty)$, acting over a suitable infinite-dimensional Hilbert space $E$. Thus, this work can be seen as a continuation of [1].

Notice that the infinite-dimensional unitary group $U(\infty)$ is one of the basic examples of big groups whose irreducible representations depend on infinitely many parameters. General principles of harmonic analysis on this group are developed by Olshanski [2].

The investigated Hardy space $H_{\chi}^{p}$ in the unitary case $p=2$ is antilinearly unitary isometric to a symmetric Fock space $F$, generated by $E$ (see Theorem 2 in [1]). Therefore, we can also apply obtained results for $H_{\chi}^{p}$ to best approximations in the symmetric Fock space F.

Now we talk briefly about the content. In the introductory Sections 2 and 3, we investigate an abstract problem for a complete quasinormed abelian group $G$, containing a dense subgroup $\mathscr{G}$ with a given continuous approximation scale. To solve this problem, we use an interpolating scale of special Besov-type subgroups $B_{\tau}^{\alpha}(\mathscr{G})$, defined by approximation $E$ functionals. Preliminary information about approximations with the help of $E$-functionals in the general case of complete quasinormed abelian groups can be found in $[3,7.1]$ and [4].
In Theorem 5 we establish a general (one of many possible) form of the Bernstein-Jackson inequalities for the considered Besov-type scale $B_{\tau}^{\alpha}(\mathscr{G})$.

The main result is in Theorem 7 that, in some sense, gives a solution of best approximation problem in the Hardy spaces $H_{\chi}^{p}$ for the case of Besov-type scale. We establish an analogue of the Bernstein-Jackson inequalities which sharply characterizes a behavior of best approximations for functions of infinitely many variables.

It should be noted that we consider the cases of linear and nonlinear approximations in the Hardy spaces $H_{\chi}^{p}$. Recall that extensive information on nonlinear approximations by discrete scales in various Banach spaces, having wide constructive implementations, can be found, for example, in DeVore [5].

Moreover, in Theorem 8 we show an application of the Bernstein-Jackson inequalities to best linear and nonlinear approximations in symmetric Fock spaces.

\section{Besov-Type Approximation Scales}

Following [4], we consider a complete quasinormed abelian group $\left(G,|\cdot|_{G}\right)$ under addition "+" with the neutral element 0 , where the quasinorm $|\cdot|_{G}$ is determined by the following assumptions:

(1) $|0|_{G}=0$ and $|a|_{G}>0$ for all nonzero $a \in G$,

(2) $|a|_{G}=|-a|_{G}$ for all $a \in G$, 
(3) $|a+b|_{G} \leq \gamma\left(|a|_{G}+|b|_{G}\right)$ for all $a, b \in G$ and some fixed $\gamma \geq 1$.

In what follows we additionally suppose that the group $G$ contains a dense subgroup

$$
\mathscr{G}=\bigcup\left\{\mathscr{G}_{t}: t>0\right\}
$$

with a continuous approximation scale of subsets $\mathscr{G}_{t}$, possessing the following properties:

(i) $\mathscr{G}_{t}=-\mathscr{G}_{t}$ for all $t \geq 0$, at that $\mathscr{G}_{0}=\{0\}$,

(ii) $\mathscr{G}_{s} \subset \mathscr{G}_{t}$ for all $0 \leq s<t$,

(iii) $\mathscr{G}_{t} \pm \mathscr{G}_{s} \subset \mathscr{G}_{\gamma(t+s)}$ for all $t, s \geq 0$.

On the subgroup $\mathscr{G}$ we define the quasinorm

$$
|a|=|a|_{G}+\inf \left\{t>0: a \in \mathscr{G}_{t}\right\}
$$

which satisfies the conditions $|a|=|-a|$ and

$$
|a+b| \leq \gamma(|a|+|b|)
$$

for all $a, b \in \mathscr{G}$ with the same constant $\gamma \geq 1$.

In fact, if we put

$$
r(a)=\inf \left\{t>0: a \in \mathscr{G}_{t}\right\}
$$

then $r(a)=r(-a)$ via property (i) and

$$
\begin{aligned}
r(a+b) & =\inf \left\{\tau>0: a+b \in \mathscr{G}_{\tau}\right\} \\
& =\gamma \inf \left\{t+s>0: a+b \in \mathscr{G}_{\gamma(t+s)}\right\} \\
& \leq \gamma \inf \left\{t+s>0: a \in \mathscr{G}_{t}, b \in \mathscr{G}_{s}\right\} \\
& \leq \gamma \max \{r(a), r(b)\} \\
& \leq \gamma[r(a)+r(b)]
\end{aligned}
$$

for all $a \in \mathscr{G}_{t}$ and $b \in \mathscr{G}_{s}$ via property (iii). As a result, $|\cdot|$ is a quasinorm with the constant $\gamma$, because the quasinorm $|\cdot|_{G}$ is the same.

So, the following contracting dense embedding holds:

$$
\mathscr{G} \leftrightarrow G .
$$

Let us endow the dense subgroup $\mathscr{G}$ with the quasinorm $|\cdot|$ and consider on the whole group $G$ the so-called approximation $E$-functional (see, e.g., $[3,7.1]$ )

$$
E(t, a)=\inf \left\{\left|a-a^{0}\right|_{G}: a^{0} \in \mathscr{G},\left|a^{0}\right|<t\right\}
$$

with $a \in G$. Given the pairs $\{0<\alpha<\infty, 0<\tau \leq \infty\}$ and $\{0 \leq \alpha<\infty, \tau=\infty\}$ we assign in $G$ the Besov-type approximation abelian subgroups

$$
B_{\tau}^{\alpha}(\mathscr{G})=\left\{a \in G:|a|_{B_{\tau}^{\alpha}}<\infty\right\}
$$

endowed with the quasinorm

$$
|a|_{B_{\tau}^{\alpha}}= \begin{cases}\left(\int_{0}^{\infty}\left[t^{\alpha} E(t, a)\right]^{\tau} \frac{d t}{t}\right)^{1 / \tau}, & \tau<\infty, \\ \sup _{t>0} t^{\alpha} E(t, a), & \tau=\infty,\end{cases}
$$

which is determined by the given subgroup $\mathscr{G}$. Notice that the function $|\cdot|_{B_{\tau}^{\alpha}}$ is a quasinorm on $B_{\tau}^{\alpha}(\mathscr{G})$ via [3, Lemma 7.1.6].
Definition 1. One calls the scale $B_{\tau}^{\alpha}(\mathscr{G})$ Besov-type.

Notice that if $G=\mathbb{R}$ then $B_{\tau}^{\alpha}(\mathscr{G})$ exactly coincides with the scale of classic Besov spaces.

For any pairs index $\{0<\vartheta<1,1 \leq p \leq \infty\}$ or $\{0<\vartheta \leq$ $1, p=\infty\}$, using the Peetre $K$-functional for the pair groups $\mathscr{G}$ and $G$,

$$
K(t, a ; \mathscr{G}, G)=\inf _{a=a^{0}+a^{1}}\left(\left|a^{0}\right|+t\left|a^{1}\right|_{G}\right), \quad t>0
$$

with $a^{0} \in \mathscr{G}$ and $a^{1} \in G$, we can define the interpolation abelian subgroup

$$
(\mathscr{G}, G)_{\vartheta, p}=\left\{a \in G:|a|_{(\mathscr{G}, G)_{\vartheta, p}}<\infty\right\}
$$

endowed with the quasinorm (see [3])

$$
|a|_{(\mathscr{G}, G)_{\vartheta, p}}= \begin{cases}\left(\int_{0}^{\infty}\left[t^{-\vartheta} K(t, a ; \mathscr{G}, G)\right]^{p} \frac{d t}{t}\right)^{1 / p}, & p<\infty \\ \sup _{0<t<\infty} t^{-\vartheta} K(t, a ; \mathscr{G}, G), & p=\infty .\end{cases}
$$

Lemma 2. Let $\left[B_{\tau}^{\alpha}(\mathscr{G})\right]^{\vartheta}$ be the subgroup $B_{\tau}^{\alpha}(\mathscr{G})$ endowed with the quasinorm $|\cdot|_{B_{\tau}^{\alpha}}^{9}$. Then the equality (up to a quasinorm equivalence)

$$
\left[B_{\tau}^{\alpha}(\mathscr{G})\right]^{\vartheta}=(\mathscr{G}, G)_{\vartheta, p}
$$

with the parameters

$$
\begin{gathered}
\vartheta=\frac{1}{\alpha+1}, \\
\tau=p \vartheta
\end{gathered}
$$

holds. As a consequence, the subgroup $B_{\tau}^{\alpha}(\mathscr{G})$ is complete and densely embedded in $G$.

Proof. The first claim immediately implies from the known [3, Theorem 7.1.7]. Now we are going to check the completeness and dense embedding. Let $\mathscr{G}+G$ be endowed with the quasinorm

$$
|a|_{\mathscr{G}+G}=\inf _{a=a^{0}+a^{1}}\left(\left|a^{0}\right|+\left|a^{1}\right|_{G}\right),
$$

where $a^{0} \in \mathscr{G}$ and $a^{1} \in G$. Since $|a| \geq|a|_{G}$ and $\mathscr{G}+G=G$, we have

$$
|a|_{\mathscr{G}+G}=|a|_{G}
$$

for all $a \in G$. Hence, the space $G$ with the quasinorm $|\cdot|_{\mathscr{G}+G}$ is complete. Therefore, every series $\sum a_{n}$ with $a_{n} \in(\mathscr{G}, G)_{\vartheta, p}$ such that $\sum\left|a_{n}\right|_{(\mathscr{G}, G)_{9, p}}<\infty$ is convergent to an element $a \in$ $G=\mathscr{G}+G$. Using the inequality

$$
\left|\sum a_{n}\right|_{(\mathscr{G}, G)_{\vartheta, p}} \leq \gamma \sum\left|a_{n}\right|_{(\mathscr{G}, G)_{9, p}}
$$

we obtain that $a \in(\mathscr{G}, G)_{\vartheta, p}$. Hence, $(\mathscr{G}, G)_{\vartheta, p}$ is complete. Isomorphism (13) implies that $\left[B_{\tau}^{\alpha}(\mathscr{G})\right]^{\vartheta}$ is complete and densely embedded in $G$. Thus, $B_{\tau}^{\alpha}(\mathscr{G})$ is complete and densely embedded as well. 
Corollary 3. If $0<\tau<\infty, 0<\vartheta<1$, and $\alpha=(1-\vartheta) \alpha_{0}+\vartheta \alpha_{1}$ with $\alpha_{0} \neq \alpha_{1}$ then

$$
\left(B_{\tau_{0}}^{\alpha_{0}}(\mathscr{G}), B_{\tau_{1}}^{\alpha_{1}}(\mathscr{G})\right)_{\vartheta, \tau}=B_{\tau}^{\alpha}(\mathscr{G})
$$

and there exist constants $c_{1}, c_{2}$ such that

$$
|a|_{B_{\tau}^{\alpha}} \leq c_{1}|a|_{B_{\tau_{0}}^{\alpha_{0}}}^{1-\vartheta}|a|_{B_{\tau_{1}}^{\alpha_{1}}}^{9}
$$

for all $a \in B_{\tau_{0}}^{\alpha_{0}}(\mathscr{G}) \cap B_{\tau_{1}}^{\alpha_{1}}(\mathscr{G})$ and

$$
K\left(t, a ; B_{\tau_{0}}^{\alpha_{0}}, B_{\tau_{1}}^{\alpha_{1}}\right) \leq c_{2} t^{\vartheta}|a|_{B_{\tau}^{\alpha}}
$$

for all $a \in B_{\tau}^{\alpha}(\mathscr{X})$, where $K\left(t, a ; B_{\tau_{0}}^{\alpha_{0}}, B_{\tau_{1}}^{\alpha_{1}}\right)$ means the Peetre K-functional of the groups $B_{\tau_{0}}^{\alpha_{0}}(\mathscr{G})$ and $B_{\tau_{1}}^{\alpha_{1}}(\mathscr{G})$.

Proof. Applying the reiteration property of real interpolation [3, Theorem 3.11.5] for the indexes

$$
\begin{gathered}
\vartheta=\frac{1}{\alpha+1}, \\
\tau=p \vartheta, \\
0<\eta<1, \\
\vartheta=(1-\eta) \vartheta_{0}+\eta \vartheta_{1}, \\
\vartheta_{i}=\frac{1}{\alpha_{i}+1}
\end{gathered}
$$

$(i=0,1)$ we obtain the following equality (clearly, up to a quasinorm equivalence):

$$
\left(\left[B_{\tau_{0}}^{\alpha_{0}}\right]^{\vartheta_{0}},\left[B_{\tau_{1}}^{\alpha_{1}}\right]^{\vartheta_{1}}\right)_{\eta, p}=\left[B_{\tau}^{\alpha}\right]^{\vartheta} .
$$

In turn, applying the interpolation degree property [3, Theorem 3.11.6], we obtain

$$
\left(\left[B_{\tau_{0}}^{\alpha_{0}}\right]^{\vartheta_{0}},\left[B_{\tau_{1}}^{\alpha_{1}}\right]^{\vartheta_{1}}\right)_{\eta, p}=\left(B_{\tau_{0}}^{\alpha_{0}}, B_{\tau_{1}}^{\alpha_{1}}\right)_{\varrho, \tau}^{\vartheta}
$$

with $\varrho=\eta \vartheta_{1} / \vartheta$. Now, equalities (22) and (23) for $\alpha=(1-$ $\varrho) \alpha_{0}+\varrho \alpha_{1}$ yield (18) with $\varrho=\vartheta$.

On the other hand, inequalities (19) and (20) are a consequence of (18) and well known property of the real interpolation [3, Theorem 3.11.2].

Corollary 4. If $0<\tau \leq \varrho<\infty$ then the following continuous embedding holds:

$$
B_{\tau}^{\alpha}(\mathscr{G}) \rightarrow B_{\varrho}^{\alpha}(\mathscr{G}) .
$$

Proof. Applying Corollary 3, we get that for every element $x \in$ $\left(B_{\tau_{0}}^{\alpha_{0}}(\mathscr{G}), B_{\tau_{1}}^{\alpha_{1}}(\mathscr{G})\right)_{\mathcal{\vartheta}, \tau}$ there exists a constant $c>0$ such that the inequalities

$$
\begin{aligned}
& |a|_{\left(B_{\tau_{0}}, B_{\tau_{1}} \alpha_{\vartheta, \varrho}\right.}^{\alpha_{1}} \\
& \quad \leq\left(\operatorname{supt}_{t>0} t^{-\vartheta} K(t, a ; \cdot)\right)^{1-\tau / \varrho}\left(\int_{0}^{\infty}\left[t^{-\vartheta} K(t, a ; \cdot)\right]^{\tau} \frac{d t}{t}\right)^{1 / \varrho} \\
& \quad \leq c|a|_{\left(B_{\tau_{0}}^{\alpha_{0}}, B_{\tau_{1}}^{\alpha_{1}}\right)_{\vartheta, \tau}}
\end{aligned}
$$

hold. Hence, the embedding

$$
\left(B_{\tau_{0}}^{\alpha_{0}}, B_{\tau_{1}}^{\alpha_{1}}\right)_{\vartheta, \tau} \rightarrow\left(B_{\tau_{0}}^{\alpha_{0}}, B_{\tau_{1}}^{\alpha_{1}}(\mathscr{G})\right)_{\vartheta, \varrho}
$$

is continuous. Finally using (18), we obtain (24).

\section{Best Approximations}

Let the subgroup $\mathscr{G}$ be endowed with the quasinorm $|\cdot|$ of form (2). Now we are going to consider the problem of best approximation of a given element in a complete abelian group $G$ by elements of a fixed subset $\mathscr{G}_{t} \subset \mathscr{G}$.

We denote the distance between $a \in G$ and a subset $\mathscr{G}_{t}$ with a fixed $t>0$ by

$$
\delta(t, a)=\inf \left\{\left|a-a^{0}\right|_{G}: a^{0} \in \mathscr{G}_{t}\right\}
$$

This distance characterizes the error of the best approximation of $a$ by elements of $\mathscr{G}_{t}$.

To investigate this problem, we will use the scale of subgroups $B_{\tau}^{\alpha}(\mathscr{G})$ determined for the pairs of indexes $\{0<$ $\alpha<\infty, 0<\tau \leq \infty\}$ or $\{0 \leq \alpha<\infty, \tau=\infty\}$.

Theorem 5. For every $\alpha, \tau$ there are constants $c_{1}$ and $c_{2}$ so that the following analogue of the Bernstein-Jackson inequalities holds:

$$
\begin{gathered}
|a|_{B_{\tau}^{\alpha}} \leq c_{1}|a|^{\alpha}|a|_{G}, \quad a \in \mathscr{G}, \\
\delta(t, a) \leq c_{2} \frac{|a|_{B_{\tau}^{\alpha}}}{t^{\alpha}}, \quad a \in B_{\tau}^{\alpha}(\mathscr{G}) .
\end{gathered}
$$

Proof. By Lemma 2 the space $\left[B_{\tau}^{\alpha}(\mathscr{G})\right]^{\vartheta}$ is interpolating between $\mathscr{G}$ and $G$. As a consequence,

$$
\mathscr{G} \subset\left[B_{\tau}^{\alpha}(\mathscr{G})\right]^{\vartheta}=(\mathscr{G}, G)_{\vartheta, p} \subset G, \quad \vartheta=\frac{1}{\alpha+1}
$$

with $\tau=p \vartheta$. Hence, applying [3, Theorem 3.11.4(b)] for some constant $c(\vartheta, p)$ we obtain

$$
|a|_{(\mathscr{G}, G)_{\vartheta, p}} \leq c|a|^{1-\vartheta}|a|_{G}^{\vartheta}, \quad a \in \mathscr{G} .
$$

This inequality together with isomorphism (13) implies that there is a constant $c_{1}(\alpha, \tau)$ such that inequality (28) is true.

Now we will prove the second inequality. By [3, Theorem 3.11.4(a)] for some constant $c^{\prime}(\vartheta, p)$ we have

$$
K(t, a ; \mathscr{G}, G) \leq c^{\prime} t^{\vartheta}|a|_{(\mathscr{G}, G)_{\vartheta, p}}, \quad a \in(\mathscr{G}, G)_{\vartheta, p} .
$$

Hence, in virtue of isomorphism (13) there is a constant $c_{0}(\alpha, \tau)$ such that

$$
K(t, a ; \mathscr{G}, G) \leq c_{0} t^{\vartheta}|a|_{B_{\tau}^{\alpha}}^{\vartheta}, \quad a \in B_{\tau}^{\alpha}(\mathscr{G}) .
$$

Following $[3,7.1]$, we introduce the functional

$$
K_{\infty}(t, a ; \mathscr{G}, G)=\inf _{a=a^{0}+a^{1}} \max \left\{\left|a^{0}\right|, t\left|a^{1}\right|_{G}\right\}
$$


with $a^{0} \in \mathscr{G}$ and $a^{1} \in G$. The inequality

$$
K_{\infty}(t, a ; \mathscr{G}, G) \leq K(t, a ; \mathscr{G}, G)
$$

yields

$$
\frac{K_{\infty}(t, a ; \mathscr{G}, G)}{t^{\vartheta}} \leq c_{0}|a|_{B_{\tau}^{\alpha}}^{9}
$$

for all $a \in B_{\tau}^{\alpha}(\mathscr{G})$.

By [3, Lemma 7.1.2] for every $t>0$ and $a \in B_{\tau}^{\alpha}(\mathscr{G})$ there exists $s>0$ such that

$$
\begin{gathered}
K_{\infty}(t, a ; \mathscr{G}, G)=s, \\
\lim _{\varepsilon \searrow s} E(\varepsilon, a):=E(s+0, a) \leq \frac{s}{t} .
\end{gathered}
$$

For any fixed $a$ the function $E(s, a)$ is decreasing in the variable $s$ (see [3, Lemma 7.1.1]). Therefore,

$$
E(s, a) \leq E\left(s_{1}+0, a\right) \leq \frac{s_{1}}{t}, \quad a \in B_{\tau}^{\alpha}(\mathscr{G})
$$

for every $s_{1}>0$ such that

$$
s_{1} \leq K_{\infty}(t, a ; \mathscr{G}, G)=s
$$

for all $a \in B_{\tau}^{\alpha}(\mathscr{G})$. It follows that

$$
[E(s, a)]^{\vartheta} \leq \frac{s_{1}^{\vartheta}}{t^{9}} \leq \frac{s^{\vartheta-1} s}{t^{\vartheta}}, \quad a \in B_{\tau}^{\alpha}(\mathscr{G}) .
$$

As a result, we obtain

$$
s^{1-9}[E(s, a)]^{9} \leq \frac{K_{\infty}(t, a ; \mathscr{G}, G)}{t^{9}}, \quad a \in B_{\tau}^{\alpha}(\mathscr{G}) .
$$

Using (36), we have

$$
s^{1-\vartheta}[E(s, a)]^{\vartheta} \leq c_{0}|a|_{B_{\tau}^{\alpha}}^{9} .
$$

Substituting $\alpha=(1-\vartheta) / \mathcal{\vartheta}$, we get

$$
s^{\alpha} E(s, a) \leq c_{0}^{1 / \vartheta}|a|_{B_{\tau}^{\alpha}}, \quad a \in B_{\tau}^{\alpha}(\mathscr{G}) .
$$

On the other hand, if

$$
\left|a^{0}\right|=r\left(a^{0}\right)+\left|a^{0}\right|_{G}<s,
$$

then $r\left(a^{0}\right)<s-\left|a^{0}\right|_{G}$, where is denoted

$$
r\left(a^{0}\right)=\inf \left\{t>0: a^{0} \in \mathscr{G}_{t}\right\} .
$$

So, $a^{0} \in \mathscr{G}_{t}$ for all numbers $t>0$ such that

$$
r\left(a^{0}\right)<t<s-\left|a^{0}\right|_{G} .
$$

The embedding $\mathscr{G}_{t} \subset \mathscr{G}_{s}$ yields $a^{0} \in \mathscr{G}_{s}$. Hence, the following inequality

$$
\delta(s, a) \leq E(s, a), \quad a \in G, s>0
$$

holds. Now taking $c_{2}=c_{0}^{1 / 9}$ in (43) and using (47), we finally obtain the required inequality (29).

\section{Hardy Spaces of Infinitely Many Variables}

We will investigate the Hardy space $H_{\chi}^{p}(0<p \leq \infty)$ of complex functions on the infinite-dimensional group

$$
U(\infty)=\bigcup\{U(m): m \in \mathbb{N}\}
$$

integrable with respect to a projective limit $\chi$ of probability Haar measures $\chi_{m}$, determined on the corresponding $m$ dimensional unitary matrix groups $U(m)$.

The measure $\chi$ is determined on, the so-called, the space of virtual unitary matrices $\mathcal{U}$, being a projective limit of $U(m)$, which was earlier studied by Neretin [6] and Olshanski [2]. The main feature of this measure is the fact that it is invariant under the right action over $\mathfrak{U}$ of the infinite-dimensional group

$$
U^{2}(\infty):=U(\infty) \times U(\infty) .
$$

The Hardy space $H_{\chi}^{p}$ in the case $p=2$ was investigated in [1]. Let us describe the space $H_{\chi}^{p}$ for all $p \in(0, \infty]$ in more detail.

Let $\mathbb{C}^{m}(m \in \mathbb{N})$ be the $m$-dimensional complex Hilbert space with the scalar product $\langle\cdot \mid \cdot\rangle_{\mathbb{C}^{m}}$ and the canonical orthonormal basis

$$
\mathscr{E}\left(\mathbb{C}^{m}\right)=\left\{\mathfrak{e}_{m 1}, \ldots, \mathfrak{e}_{m m}\right\},
$$

where $\mathbf{e}_{m l}=\underbrace{(\overbrace{0, \ldots, 0,1}^{l}, 0, \ldots, 0)_{m}}$. Consider the Hilbertian sum with the scalar product $\langle x \mid y\rangle_{\mathrm{E}}=\sum_{m}\left\langle x_{m} \mid y_{m}\right\rangle_{\mathbb{C}^{m}}$,

$$
\begin{aligned}
\mathrm{E} & :=\bigoplus_{m \in \mathbb{N}} \mathrm{E}_{m} \\
& =\left\{x=\left(x_{m}\right): x_{m} \in \mathbb{C}^{m},\|x\|_{\mathrm{E}}=\left(\sum_{m \in \mathbb{N}}\left\|x_{m}\right\|_{\mathbb{C}^{m}}^{2}\right)^{1 / 2}<\infty\right\},
\end{aligned}
$$

where every coordinate $x_{m} \in \mathbb{C}^{m}$ is identified with its image $\left(0, \ldots, 0, x_{m}, 0, \ldots\right) \in \mathrm{E}_{m}$ under the natural embedding $\mathbb{C}^{m} \rightarrow \mathrm{E}$. Then the system

$$
\mathscr{E}\left(\mathrm{E}_{m}\right)=\left\{\mathfrak{e}_{m 1}, \ldots, \boldsymbol{e}_{m m}\right\}
$$

with $\mathfrak{e}_{m l} \sim(\overbrace{0, \ldots, 0, \mathfrak{e}_{m l}}^{m+l}, 0, \ldots) \in \mathrm{E}_{m},(l=1, \ldots, m)$ forms the canonical orthonormal basis in $\mathrm{E}_{m}$ and the canonical orthonormal basis of $E$ has the form

$$
\mathscr{E}(\mathrm{E})=\bigcup\left\{\mathscr{E}\left(\mathrm{E}_{m}\right): m \in \mathbb{N}\right\} .
$$

Let $U(m)$ be the group of unitary $(m \times m)$-matrices with the unit $\mathbb{1}_{m}$. We equip every group $U(m)$ with the probability Haar measure $\chi_{m}$. The right action of the cartesian product

$$
U^{2}(m):=U(m) \times U(m)
$$

over the group $U(m)$, we define as follows

$$
u \cdot g=w^{-1} u v, \quad g=(v, w) \in U^{2}(m)
$$

for all $u, v, w \in U(m)$. 
We write every matrix $u_{m} \in U(m)$ with $n>m$ in the block matrix form

$$
u_{n}=\left[\begin{array}{cc}
u_{n-m} & a \\
b & t
\end{array}\right]
$$

where $u_{n-m} \in U(n-m)$, corresponding to the matrix partition

$$
n \searrow n=(n-m)+m \searrow(n-m)+m \text {. }
$$

In [6, Proposition 0.1], [2, Lemma 3.1] it was proven that the Livšic-type mapping $\pi_{m}^{n}: U(n) \rightarrow U(n-m)$

$$
\begin{aligned}
\pi_{m}^{n}: u_{n} & =\left[\begin{array}{cc}
u_{n-m} & a \\
b & t
\end{array}\right] \longmapsto u_{m} \\
& = \begin{cases}u_{n-m}-a(1+t)^{-1} b, & t \neq-\mathbb{1}_{m} \\
u_{n-m}, & t=-\mathbb{1}_{m}\end{cases}
\end{aligned}
$$

(which is not a group homomorphism) is Borel and surjective onto $U(n-m)$ which commutes with the right action of $U^{2}(n-$ $m)$.

As is known [6, Theorem 1.6], the pullback of the probability Haar measure $\chi_{m}$ on $U(m)$ under the projections $\pi_{m}^{n}$ is the probability Haar measure $\chi_{n}$ on $U(n)$, that is,

$$
\chi_{m} \circ \pi_{m}^{n}=\chi_{n}, \quad n>m .
$$

Consider the projective limits

$$
\mathcal{U}=\lim _{\longleftarrow} U(m),
$$

taken with respect to the surjective Borel projections $\pi_{m}^{n}$. The canonical projection

$$
\pi_{m}: \mathfrak{U} \longrightarrow U(m)
$$

such that $\pi_{m}=\pi_{m}^{n} \circ \pi_{n}$ are surjective by surjectivity of $\pi_{m}^{n}$. The virtual unitary group $\mathfrak{U}$ acts isometrically over the Hilbert space $\mathrm{E}$ by coordinate-wise way,

$$
u(x)=\left(\left[\pi_{m}(u)\right]\left(x_{m}\right)\right)_{m \in \mathbb{N}} \in \mathrm{E}
$$

with $\left[\pi_{m}(u)\right]\left(x_{m}\right) \in \mathrm{E}_{m}$, for all elements $u \in \mathcal{U}$ and $x=$ $\left(x_{m}\right) \in \mathrm{E}$.

Following $[2,6]$ with the help of the Kolmogorov consistent theorem, we uniquely define a probability measure $\chi$ on $\mathcal{U}$, as the projective limit under mapping (59),

$$
\chi=\lim _{\longleftarrow} \chi_{m}
$$

which satisfies the equality

$$
\chi=\chi_{m} \circ \pi_{m}, \quad m \in \mathbb{N} .
$$

The right action of the infinite-dimensional unitary matrix groups $U^{2}(\infty)$ over the spaces of virtual unitary matrices $\mathfrak{U}$ is defined (see [2, Definition 4.5]) as

$$
\pi_{m}(u \cdot g)=w^{-1} \pi_{m}(u) v, \quad u \in \mathfrak{U}
$$

where $m$ is so large that $g=(v, w) \in U^{2}(m)$. The measure $\chi$ is invariant under the right actions (65). Moreover, $\chi$ is a Radon measure (see [1, Lemma 1]).

A complex function on $\mathfrak{U}$ is called cylindrical [2, Definition 4.5] if it has the form

$$
f(u)=\left(f_{m} \circ \pi_{m}\right)(u), \quad u \in \mathfrak{U}
$$

for a certain $m \in \mathbb{N}$ and complex function $f_{m}$ on $U(m)$. Any such function $f$ is $\chi$-essentially bounded.

Let $L_{\chi}^{\infty}$ denote the closure of the algebraic hull of all cylindrical $\chi$-essentially bounded functions (72) with respect to the norm

$$
\|f\|_{L_{\chi}^{\infty}}=\underset{u \in \mathfrak{U}}{\operatorname{ess} \sup }|f(u)|
$$

Let $L_{\chi}^{p}(0<p<\infty)$ be the space of $\chi$-integrable complex functions $f$ on the space of virtual matrices $\mathfrak{U}$ with the finite quasinorm $\|f\|_{L_{\chi}^{p}}=\left(\int_{\mathfrak{U}}|f|^{p} d \chi\right)^{1 / p}$ which is a quasinormed complete additive group (see [3, Lemma 3.10.3]). Since $\chi$ is a probability measure, the contractive embedding $L_{\chi}^{\infty} \rightarrow L_{\chi}^{p}$ holds and

$$
\|f\|_{L_{\chi}^{p}} \leq\|f\|_{L_{\chi}^{\infty}}, \quad f \in L_{\chi}^{\infty} .
$$

As is well known [3], the constant $\gamma$ in the triangle inequality

$$
\|f+g\|_{L_{\chi}^{p}} \leq \gamma\left(\|f\|_{L_{\chi}^{p}}+\|g\|_{L_{\chi}^{p}}\right)
$$

may be chosen equal to 1 for the case $1 \leq p \leq \infty$ and $2^{(1-p) / p}$ for the case $0<p<1$.

Let us denote

$$
\begin{gathered}
(m):=(m 1, \ldots, m m), \\
k_{(m)}:=\left(k_{m 1}, \ldots, k_{m m}\right) \in \mathbb{Z}_{+}^{m}, \\
\left|k_{(m)}\right|:=k_{m 1}+\cdots+k_{m m}, \\
k_{(m)} !:=k_{m 1} ! \cdot \ldots \cdot k_{m m} ! \\
{[m]:=\{(11),(21,22), \ldots,(m 1, \ldots, m m)\} .}
\end{gathered}
$$

Consider the system of all finite sequences

$$
\{k\}:=\left\{k_{(1)}, \ldots, k_{(r)}, \ldots\right\} \in \bigcup_{r=1}^{\infty} \mathbb{Z}_{+}^{r}
$$

and let $m$ and $\operatorname{supp}\{k\}$ denote, respectively, the largest index $r$ and the set of all indexes $r$ in the sequence $\{k\}$ so that the integer vector $k_{(r)}=\left(k_{r 1}, \ldots, k_{r r}\right)$ is nonzero. Take the corresponding system of $\chi_{m}$-integrable cylindrical functions of the variable $u \in \mathfrak{U}$,

$$
\begin{gathered}
\mathbf{e}_{[m]}^{*\{k\}}:=\mathbf{e}_{(1)}^{* k_{(1)}} \cdot \ldots \cdot \mathbf{e}_{(m)}^{* k_{(m)}}, \\
\mathbf{e}_{(r)}^{* k_{(r)}}(u)=\prod_{i=1}^{r}\left\langle\left(\pi_{r} \circ u\right)\left(\mathbf{e}_{r 1}\right) \mid \mathfrak{e}_{r i}\right\rangle_{\mathrm{E}}^{k_{r i}} .
\end{gathered}
$$


For any $p \in(0, \infty)$ equality $(64)$ yields

$$
\begin{aligned}
\left\|\mathfrak{e}_{[m]}^{*\{k\}}\right\|_{L_{\chi m}^{p}}^{p} & =\int_{U(m)}\left|\mathfrak{e}_{[m]}^{*\{k\}} \circ \pi_{m}^{-1}\right|^{p} d \chi_{m} \\
& =\int_{\mathfrak{U}}\left|\mathfrak{e}_{[m]}^{*\{k\}}\right|^{p} d \chi=\left\|\mathfrak{e}_{[m]}^{*\{k\}}\right\|_{L_{\chi}^{p}}^{p} .
\end{aligned}
$$

Similarly, we have in the case $p=\infty$. Let us form the systems of normalized cylindrical functions

$$
\begin{gathered}
\mathscr{E}_{m}^{*}=\left\{\left\|\mathfrak{e}_{[m]}^{*\{k\}}\right\|_{L_{\chi}^{p}}^{-1} \mathfrak{e}_{[m]}^{*\{k\}}:\{k\} \in \chi_{r=1}^{m} \mathbb{Z}_{+}^{r}\right\}, \\
\mathscr{E}^{*}=\left\{\mathscr{E}_{m}^{*}: m \in \mathbb{N}\right\}
\end{gathered}
$$

with fixed and nonfixed $m$. Clearly (see [1]), they belong to the space $L_{\chi}^{\infty}$.

Definition 6. The Hardy space $H_{\chi}^{p}(0<p \leq \infty)$ on the space of virtual unitary matrices $\mathcal{U}$ is defined as $L_{\chi}^{p}$-closure of the complex linear span of $\mathscr{E}^{*}$ (for $p=2$ see [1, Definition 5]).

It is essential to note that the system $\mathscr{E}^{*}$ forms an orthonormal basis in the Hilbert space $H_{\chi}^{p}$ in the case $p=2$ [1, Theorem 1].

\section{Approximations in Hardy Spaces}

Now, we will consider a quasinormed group $G$, as an additive subgroup in the Hardy space $H_{\chi}^{p}(0<p \leq \infty)$ endowed with the quasinorm $\|\cdot\|_{L_{\chi}^{p}}$. We will analyze three cases of approximations to a linear and nonlinear setting.

(I) For the first case of linear approximation, we use the linear span in the space $H_{\chi}^{p}$ of all cylindrical functions $\left\{\mathscr{E}_{m}^{*}: m \leq t\right\}$ of not greater than $t$ first variables, that is,

$$
\mathscr{G}_{t}=\operatorname{span}\left\{\mathscr{E}_{m}^{*}: m \leq t\right\}
$$

It corresponds to the approximation of functions with infinite-dimensional variables by functions of fixed finite number variables.

(II) For the second case of linear approximation, we use the linear combinations in $H_{\chi}^{p}$ of all cylindrical functions $\mathscr{E}^{*}$ such that the number

$$
|\{k\}|:=\sum_{r \in \operatorname{supp}\{k\}}\left|k_{(r)}\right|
$$

is not greater than $t$; that is, we choose

$$
\mathscr{G}_{t}=\operatorname{span}\left\{\boldsymbol{e}_{[m]}^{*\{k\}}:|\{k\}| \leq t, m \in \mathbb{N}\right\} .
$$

It corresponds to the approximation of functions by polynomials of a fixed finite degree.
(III) For nonlinear approximation, we use all not greater than $t$-terms linear combinations in $H_{\chi}^{p}$ of cylindrical functions from $\mathscr{E}^{*}$; that is, we choose

$$
\mathscr{G}_{t}=\bigcup_{\{k\}} \operatorname{span}\left\{\boldsymbol{e}_{[m]}^{*\{k\}}: \# \operatorname{supp}\{k\} \leq t\right\},
$$

where \# means cardinality of a set. Notice that, in contrast to linear approximation, the set $\mathscr{G}_{t}$ is not linear. A sum of two elements in $\mathscr{G}_{t}$ will in general need $2 t$ terms in its representation by $\mathscr{E}^{*}$.

In all three considered cases for any constant $\gamma \geq 1$ the embedding $\mathscr{G}_{t} \pm \mathscr{G}_{s} \subset \mathscr{G}_{\gamma(t+s)}$ holds for all $t, s \geq 0$. Therefore, we choose the constant $\gamma$ so that the Hardy space $H_{\chi}^{p}$ had to be a complete quasinormed additive subgroup. Namely, in what follows we put

$$
\gamma= \begin{cases}1, & 1 \leq p \leq \infty \\ 2^{(1-p) / p}, & 0<p<1\end{cases}
$$

Moreover, since $\{k\}$ runs over all finite subsequences in $\chi_{r=1}^{\infty} \mathbb{Z}_{+}^{r}$, the additive subgroups $\bigcup\left\{\mathscr{G}_{t}: t>0\right\}$ are total in $H_{\chi}^{p}$.

We endowed the additive subgroups

$$
\mathscr{G}=\bigcup\left\{\mathscr{G}_{t}: t>0\right\} \subset H_{\chi}^{p},
$$

corresponding to each of the cases (I)-(III) of linear and nonlinear approximations, with the quasinorms

$$
|f|=\|f\|_{L_{\chi}^{p}}+\inf \left\{t>0: f \in \mathscr{G}_{t}\right\}
$$

of form (2) with a suitable (to choice of $p$ ) constant $\gamma \geq 1$. In all considered cases for every pair index $\{0<\alpha<\infty$, $0<\tau \leq \infty\}$ or $\{0 \leq \alpha<\infty, \tau=\infty\}$ and an index $p \in(0, \infty]$ the additive subgroups

$$
B_{\tau}^{\alpha}(\mathscr{G}) \subset H_{\chi}^{p}
$$

will be denoted by $B_{\tau, p}^{\alpha}(\mathscr{G})$ and endowed with the quasinorm $|\cdot|_{B_{\tau, p}^{\alpha}}$ of form (9).

Theorem 7. (i) For every $\alpha, \tau$ the additive subgroup $B_{\tau, p}^{\alpha}(\mathscr{G})$ is complete under quasinorm (81) and the union $\bigcup_{\alpha, \tau} B_{\tau, p}^{\alpha}(\mathscr{G})$ is total in $H_{\chi}^{p}$.

(ii) For every $\alpha$, $\tau$ there are constants $c_{1}, c_{2}$ such that

$$
\inf \left\{\left\|f-f^{0}\right\|_{L_{\chi}^{p}}: f^{0} \in \mathscr{G}_{t}\right\} \leq c_{2} \frac{|f|_{B_{\tau, p}^{\alpha}}}{t^{\alpha}}
$$

for all $f \in B_{\tau, p}^{\alpha}(\mathscr{G})$ and

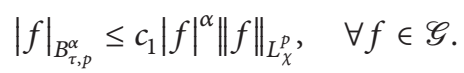

Proof. Reasoning is based on the previous auxiliary statements. The groups $\mathscr{G}$ are total in the space $H_{\chi}^{p}$ for any index $p \in(0, \infty]$ by their definitions for all three cases. Therefore, claim (i) follows from Lemma 2. 
To prove assertion (ii), we can apply Theorem 5. Namely, inequality (28) instantly implies estimation (83) for the distance from an element $x \in B_{\tau, p}^{\alpha}(\mathscr{G})$ to the additive subgroup $\mathscr{G}_{t} \subset H_{\chi}^{p}$, while inequality (29) implies estimation (84).

\section{Applications to Symmetric Fock Spaces}

Show one useful application to the theory of quantum systems. For this purpose we use the symmetric Fock spaces and their finite-dimensional subspaces.

Let $\otimes_{\mathfrak{h}}^{n} E$ stand for the complete $n$th tensor power of a Hilbert space $E$, endowed with the scalar product

$$
\left\langle x_{1} \otimes \cdots \otimes x_{n} \mid \psi_{n}\right\rangle_{\otimes_{\mathfrak{h}}^{n} \mathrm{E}}=\sum_{j}\left\langle x_{1} \mid y_{1 j}\right\rangle_{\mathrm{E}} \cdots\left\langle x_{n} \mid y_{n j}\right\rangle_{\mathrm{E}}
$$

and the norm

$$
\left\|\psi_{n}\right\|_{\otimes_{\mathfrak{h}}^{n} \mathrm{E}}=\left\langle\psi_{n} \mid \psi_{n}\right\rangle_{\otimes_{\mathfrak{h}}^{n} \mathrm{E}}^{1 / 2},
$$

where $x_{1} \otimes \cdots \otimes x_{n}, y_{1 j} \otimes \cdots \otimes y_{n j} \in \otimes_{\mathfrak{h}}^{n} \mathrm{E}$ with $x_{t j}, y_{t j} \in \mathrm{E}$ for all $t=1, \ldots, n$ and finite sums $\psi_{n}=\sum_{j} y_{1 j} \otimes \cdots \otimes y_{n j}$. Put $\otimes_{\mathfrak{h}}^{0} \mathrm{E}=\mathbb{C}$. Use the denotation

$$
x^{\otimes n}=x \otimes \cdots \otimes x, \quad x \in \mathrm{E} .
$$

Replacing $E$ by the subspace $E_{m}$, we similarly define the tensor product $\otimes_{\mathfrak{h}}^{n} \mathrm{E}_{m}$. There is the isometric embedding

$$
\otimes_{\mathfrak{h}}^{n} \mathrm{E}_{m} \rightarrow \otimes_{\mathfrak{h}}^{n} \mathrm{E} .
$$

If $m=1$ then $\otimes_{\mathfrak{h}}^{n} \mathbb{C}=\mathbb{C}$.

If $\sigma:\{1, \ldots, n\} \mapsto\{\sigma(1), \ldots, \sigma(n)\}$ runs over all $n$ elements permutations $\mathfrak{S}(n)$ then the symmetric $n$th tensor power $\odot_{\mathfrak{h}}^{n} \mathrm{E}_{m}$ is defined to be a codomain of the symmetrization mapping

$$
\begin{gathered}
\otimes_{\mathfrak{h}}^{n} \mathrm{E}_{m} \ni x_{1} \otimes \cdots \otimes x_{n} \longmapsto x_{1} \odot \cdots \odot x_{n}, \\
x_{1} \odot \cdots \odot x_{n}=\frac{1}{n !} \sum_{\sigma \in \mathbb{\subseteq}(n)} x_{\sigma(1)} \otimes \cdots \otimes x_{\sigma(n)},
\end{gathered}
$$

which is an orthogonal projector. Similarly, the symmetric $n$th tensor power $\odot_{\mathfrak{h}}^{n} \mathrm{E}$ can be defined. Clearly, $\odot_{\mathfrak{h}}^{n} \mathrm{E}_{m}$ is a closed subspace in $\odot_{\mathfrak{h}}^{n} \mathrm{E}$. Moreover, the following isometric embedding holds:

$$
\odot_{\mathfrak{h}}^{n} \mathrm{E}_{m} \rightarrow \odot_{\mathfrak{h}}^{n} \mathrm{E} .
$$

Consider the symmetric Fock space $F$ and its closed subspaces $F_{m}$ of the forms

$$
\begin{gathered}
\mathrm{F}:=\mathbb{C} \oplus \mathrm{E} \oplus\left(\odot_{\mathfrak{h}}^{2} \mathrm{E}\right) \oplus\left(\odot_{\mathfrak{h}}^{3} \mathrm{E}\right) \oplus \cdots, \\
\mathrm{F}_{m}:=\mathbb{C} \oplus \mathrm{E}_{m} \oplus\left(\odot_{\mathfrak{h}}^{2} \mathrm{E}_{m}\right) \oplus\left(\odot_{\mathfrak{h}}^{3} \mathrm{E}_{m}\right) \oplus \cdots .
\end{gathered}
$$

As is well known (see, e.g., [7]), the system of normalized symmetric tensor elements

$$
\begin{gathered}
\mathscr{E}\left(\odot_{\mathfrak{h}}^{n} \mathrm{E}_{m}\right)=\left\{\left\|\boldsymbol{e}_{(m)}^{\otimes(k)_{m}}\right\|_{F_{m}}^{-1} \mathbf{e}_{(m)}^{\otimes k_{(m)}}:\left|k_{(m)}\right|=n\right\} \\
\mathfrak{e}_{(m)}^{\otimes k_{(m)}}:=\mathbf{e}_{m 1}^{\otimes k_{m 1}} \odot \cdots \odot \mathfrak{e}_{m m}^{\otimes k_{m m}}, \\
\left\|\mathbf{e}_{(m)}^{\otimes(k)_{m}}\right\|_{F_{m}}^{2}=\frac{(k)_{m} !}{\left|(k)_{m}\right| !},
\end{gathered}
$$

indexed by the set $k_{(m)} \in \mathbb{Z}_{+}^{m}$, forms an orthonormal basis in the closed subspace $\odot_{\mathfrak{h}}^{n} \mathrm{E}_{m} \subset \mathrm{F}_{m}$.

Similarly, the system of symmetric tensor elements

$$
\begin{gathered}
\mathscr{E}\left(\odot_{\mathfrak{h}}^{n} \mathrm{E}\right)=\left\{\left\|\boldsymbol{e}_{[m]}^{\otimes\{k\}}\right\|_{\mathrm{F}}^{-1} \mathbf{e}_{[m]}^{\otimes\{k\}}:|\{k\}|=n\right\} \\
\mathfrak{e}_{[m]}^{\otimes\{k\}}:=\mathfrak{e}_{(1)}^{\otimes k_{(1)}} \odot \cdots \odot \mathfrak{e}_{(m)}^{\otimes k_{(m)}}, \\
\left\|\mathfrak{e}_{[m]}^{\otimes\{k\}}\right\|_{\mathrm{F}}^{2}=\prod_{r=1}^{m} \frac{(k)_{r} !}{(k)_{r} \mid !},
\end{gathered}
$$

indexed by finite sequences $\{k\} \in X_{r=1}^{\infty} \mathbb{Z}_{+}^{r}$, forms an orthonormal basis in the closed subspace $\odot_{\mathfrak{h}}^{n} \mathrm{E} \subset \mathrm{F}$.

In [1, Lemma 3 and Theorem 2] it is proved that in the antilinear isometric isomorphisms

$$
\begin{aligned}
\mathrm{F}_{m} & \simeq H_{\chi_{m}}^{2}, \\
\mathrm{~F} & \simeq H_{\chi}^{2}
\end{aligned}
$$

hold, where $H_{\chi_{m}}^{2}$ means the classic unitary Hardy space of analytic complex functions on the open unit ball $\mathbb{B}^{m}=$ $\left\{x_{m} \in \mathbb{C}^{m}:\left\|x_{m}\right\|_{\mathbb{C}^{m}}<1\right\}$ (see [8, Theorem 5.6.8]). It also follows that the system of cylindrical functions $\mathscr{E}_{m}^{*}$ forms an orthogonal basis in $H_{\chi_{m}}^{2}$.

On the other hand, the system of normalized symmetric tensor elements

$$
\mathscr{E}\left(\mathrm{F}_{m}\right)=\bigcup\left\{\mathscr{E}\left(\odot_{\mathfrak{h}}^{n} \mathrm{E}_{m}\right): n \in \mathbb{Z}_{+}\right\}
$$

uniquely defines the corresponding system $\mathscr{E}_{m}^{*}$ of $\chi$ integrable normalized cylindrical functions (72), because the equalities

$$
\begin{aligned}
\mathbf{e}_{(r)}^{* k_{(r)}}(u) & =\prod_{i=1}^{r}\left\langle\left(\pi_{r} \circ u\right)\left(\mathfrak{e}_{r 1}\right) \mid \mathfrak{e}_{r i}\right\rangle_{\mathrm{E}}^{k_{r i}} \\
& =\left\langle\left[\pi_{m}^{\otimes\left|k_{(r)}\right|}(u)\right]\left(\mathbf{e}_{m 1}^{\otimes\left|k_{(r)}\right|}\right) \mid \mathbf{e}_{(m)}^{\otimes k_{(r)}}\right\rangle_{\otimes_{\mathfrak{h}}^{n} \mathbb{C}^{m}}
\end{aligned}
$$

for any $r=1, \ldots, m$ hold. Moreover, their norms are completely determined by indices $\{k\}$, namely,

$$
\left\|\mathbf{e}_{[m]}^{*\{k\}}\right\|_{L_{\chi}^{2}}^{2}=\prod_{r=1}^{m} \frac{(r-1) !(k)_{r} !}{\left(r-1+\left|(k)_{r}\right|\right) !} .
$$

Thus (see [1, Theorem 2]), the above-mentioned antilinear isometries (94) and the isometric embedding $\mathrm{F}_{m} \leftrightarrow H_{\chi}^{2}$ 
are uniquely defined by the one-to-one correspondence of normalized basic elements

$$
\frac{\boldsymbol{e}_{[m]}^{\otimes\{k\}}}{\left\|\boldsymbol{e}_{[m]}^{\otimes\{k\}}\right\|_{\mathrm{F}}} \rightleftarrows \frac{\boldsymbol{e}_{[m]}^{*\{k\}}}{\left\|\boldsymbol{e}_{[m]}^{*\{k\}}\right\|_{L_{\chi}^{2}}} .
$$

Now, let us use correspondence (98) to construct approximating scales $\mathscr{G}_{t}$ in the Fock space F, which are isometrically equivalent to similar scales in the Hardy space $H_{\chi}^{2}$.

(I) Let first $\mathscr{G}_{t}$ be the linear span in the symmetric Fock space $F$ of all symmetric tensor elements $\left\{\mathscr{E}\left(\mathrm{F}_{m}\right): m \leq t\right\}$, generated by all finite-dimensional spaces $\mathrm{E}_{m}$ of not greater than $t$ dimensions; that is, we choose

$$
\mathscr{G}_{t}=\operatorname{span}\left\{\mathscr{E}\left(\mathrm{F}_{m}\right): m \leq t\right\}
$$

(II) For the second case of linear approximation, we will choose

$$
\mathscr{G}_{t}=\operatorname{span}\left\{\mathfrak{e}_{[m]}^{\otimes\{k\}}:|\{k\}| \leq t\right\} .
$$

(III) For nonlinear approximation, we will choose

$$
\mathscr{G}_{t}=\bigcup_{\{k\}} \operatorname{span}\left\{\boldsymbol{e}_{[m]}^{\otimes\{k\}}: \# \operatorname{supp}\{k\} \leq t\right\} .
$$

In all cases (I)-(III) we endowed the corresponding additive subgroups in the symmetric Fock space

$$
\mathscr{G}=\bigcup\left\{\mathscr{G}_{t}: t>0\right\} \subset \mathrm{F}
$$

with the quasinorms

$$
|\psi|=\|\psi\|_{\mathrm{F}}+\inf \left\{t>0: \psi \in \mathscr{G}_{t}\right\} .
$$

Let us denote by $B_{\tau, 2}^{\alpha}(\mathscr{G})$ and endow with the quasinorms $|\cdot|_{B_{\tau, 2}^{\alpha}}$ of the form (9) all three appropriate to the cases (I)-(III) additive subgroups

$$
B_{\tau}^{\alpha}(\mathscr{G}) \subset \mathrm{F}
$$

for every pair index $\{0<\alpha<\infty, 0<\tau \leq \infty\}$ or $\{0 \leq \alpha<$ $\infty, \tau=\infty\}$ and the fixed index $p=2$.

Using the isometric equalities (94) between the Hardy space $H_{\chi}^{2}$ and the symmetric Fock space $F$, as well as the oneto-one correspondence (98) between their normalized basic elements, we conclude that the corresponding quasinormed subgroups $B_{\tau, 2}^{\alpha}(\mathscr{G})$ in the spaces $H_{\chi}^{2}$ and $\mathrm{F}$ are isometric. So, Theorem 7 can be rewritten in the following form.

Theorem 8. (i) For every $\alpha, \tau$ the additive subgroup $B_{\tau, 2}^{\alpha}(\mathscr{G})$ is complete under the quasinorm $|\cdot|_{B_{\tau, 2}^{\alpha}}$ and the union $\bigcup_{\alpha, \tau} B_{\tau, 2}^{\alpha}(\mathscr{G})$ is total in $\mathrm{F}$.

(ii) For every $\alpha$, $\tau$ there are constants $c_{1}, c_{2}$ such that

$$
\inf \left\{\left\|\psi-\psi^{0}\right\|_{\mathrm{F}}: \psi^{0} \in \mathscr{G}_{t}\right\} \leq c_{2} \frac{|f|_{B_{\tau, 2}^{\alpha}}}{t^{\alpha}}
$$

for all $\psi \in B_{\tau, 2}^{\alpha}(\mathscr{G})$ and

$$
|\psi|_{B_{\tau, 2}^{\alpha}} \leq c_{1}|\psi|^{\alpha}\|\psi\|_{\mathrm{F}}, \quad \forall \psi \in \mathscr{G} .
$$

\section{Conflict of Interests}

The author declares that there is no conflict of interests regarding the publication of this paper.

\section{Acknowledgment}

This work was partially supported by the Center for Innovation and Transfer of Natural Sciences and Engineering Knowledge at the University of Rzeszów.

\section{References}

[1] O. Lopushansky, "Hardy-type space associated with an infinitedimensional unitary matrix group," Abstract and Applied Analysis, vol. 2013, Article ID 810735, 7 pages, 2013.

[2] G. Olshanski, "The problem of harmonic analysis on the infinite-dimensional unitary group," Journal of Functional Analysis, vol. 205, no. 2, pp. 464-524, 2003.

[3] J. Bergh and J. Lofstrom, Interpolation Spaces, Springer, Berlin, Germany, 1976.

[4] J. Peetre and G. Sparr, "Interpolation of normed abelian groups," Annali di Matematica Pura ed Applicata, vol. 92, no. 1, pp. 217262, 1972.

[5] R. DeVore, "Nonlinear approximation," Acta Numerica, vol. 7, pp. 51-150, 1998.

[6] Y. A. Neretin, "Hua-type integrals over unitary groups and over projective limits of unitary groups," Duke Mathematical Journal, vol. 114, no. 2, pp. 239-266, 2002.

[7] M. Reed and B. Simon, Methods of Modern Mathematical Physics, Vol. II, Academic Press, New York, NY, USA, 1975.

[8] W. Rudin, Function Theory in the Unit Ball of Cn, Springer, Berlin, Germany, 1980. 


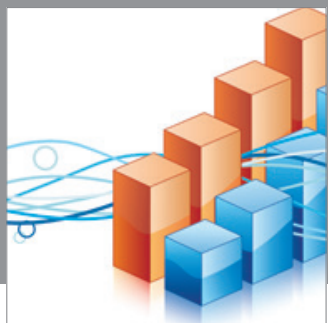

Advances in

Operations Research

mansans

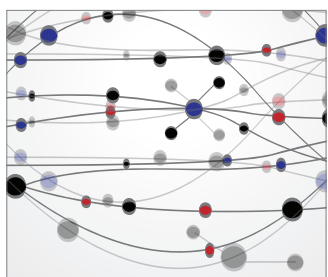

The Scientific World Journal
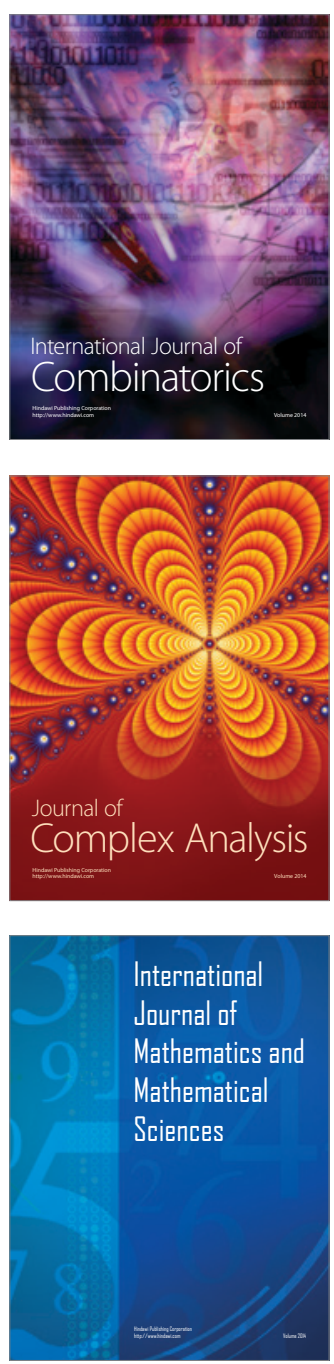
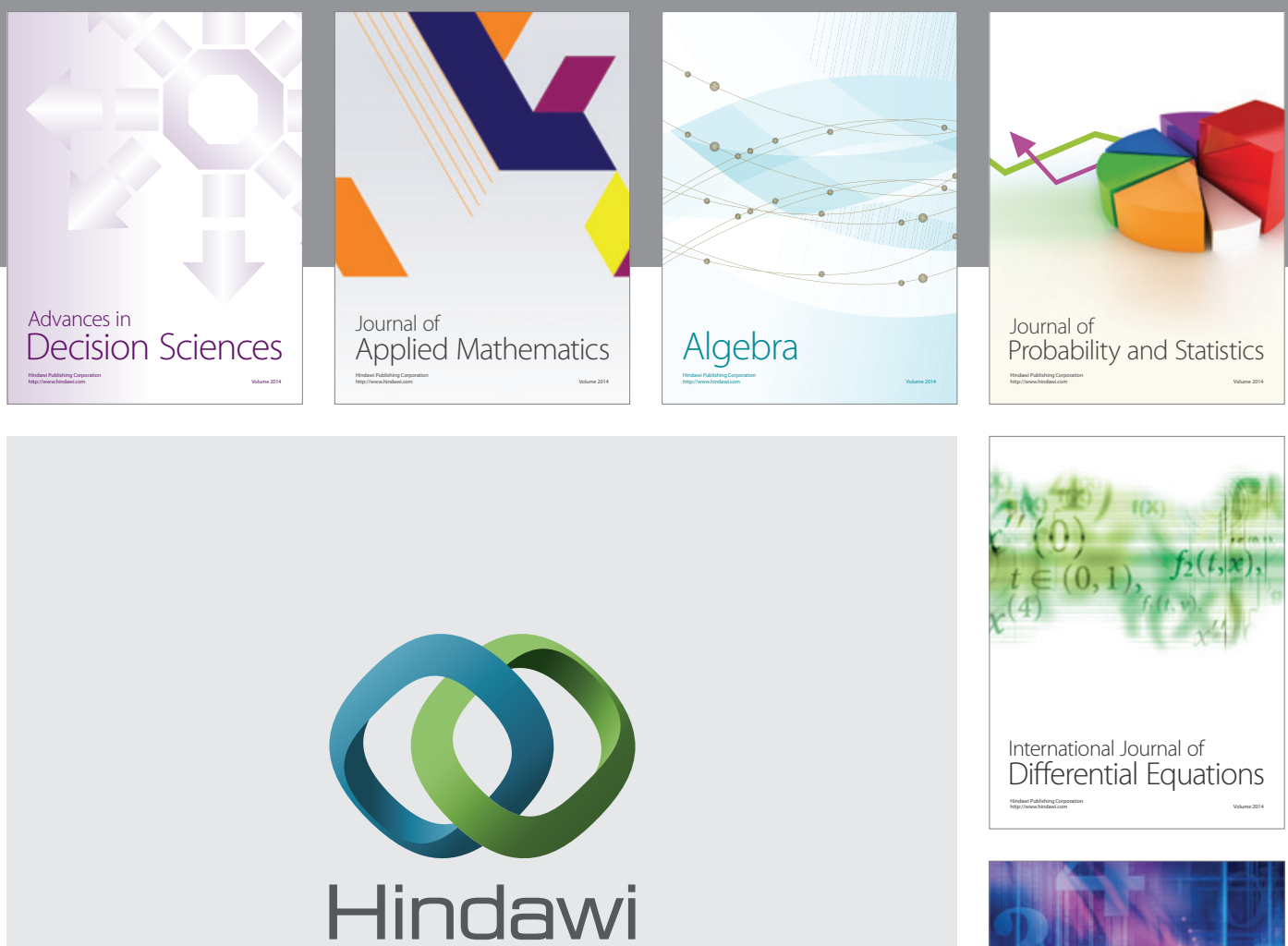

Submit your manuscripts at http://www.hindawi.com
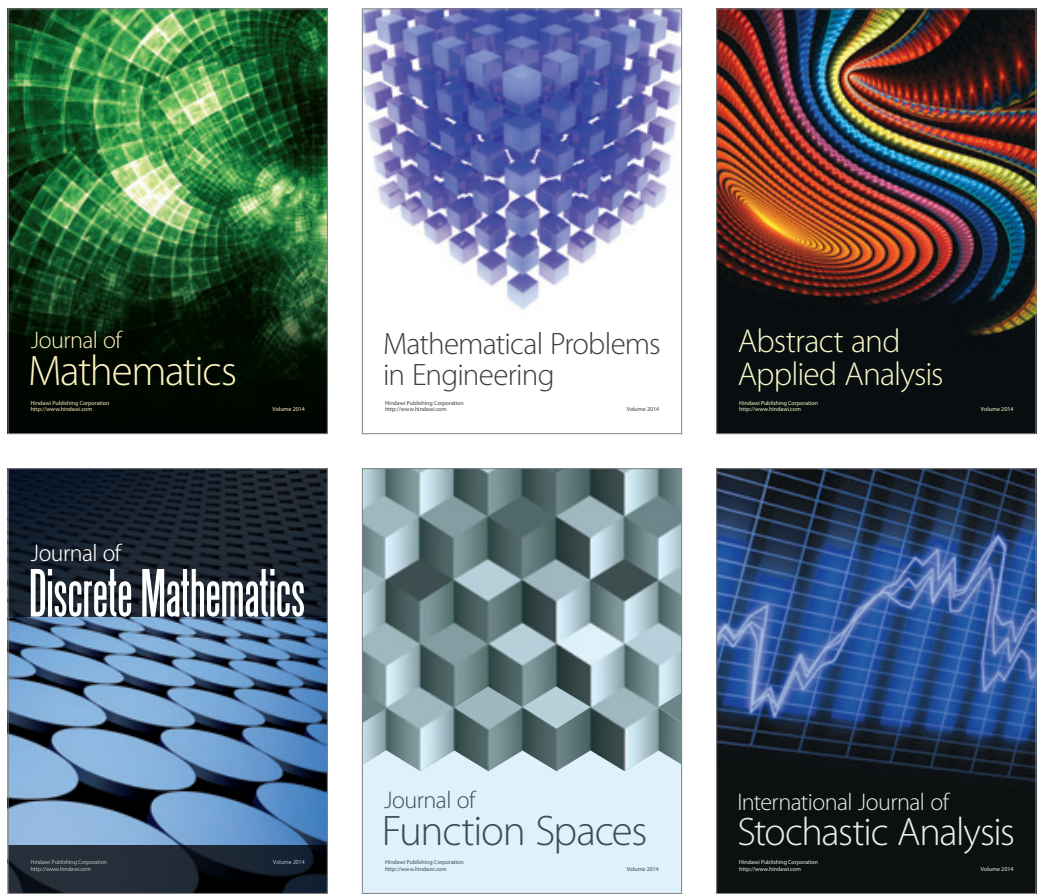

Journal of

Function Spaces

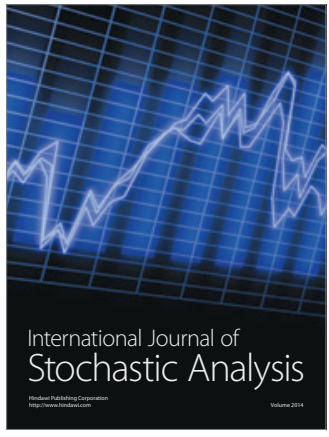

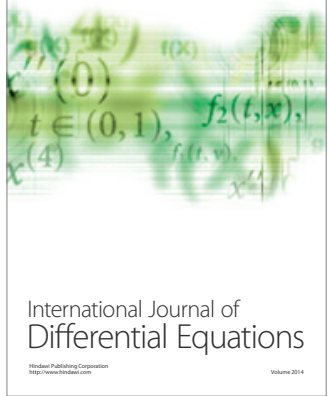
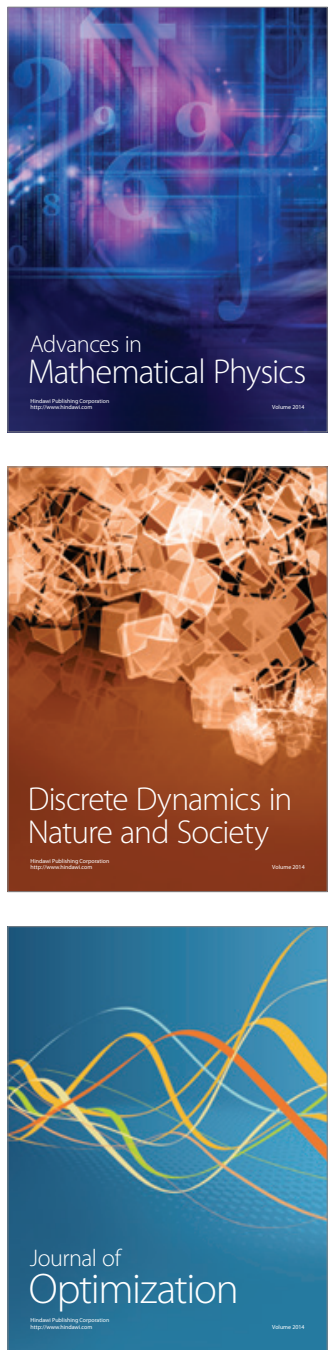